\title{
miR-214 protects erythroid cells against oxidative stress by targeting ATF4 and EZH2
}

\author{
Ming Gao ${ }^{a}$, Yun Liu ${ }^{\mathrm{a}, \mathrm{b}}$, Yue Chen ${ }^{\mathrm{a}, \mathrm{c}}$, Chunyang Yin ${ }^{\mathrm{a}}$, Jane-Jane Chen ${ }^{\mathrm{d}}$, Sijin Liu ${ }^{\mathrm{a}, *}$ \\ a State Key Laboratory of Environmental Chemistry and Ecotoxicology, Research Center for Eco-Environmental Sciences, Chinese Academy of Sciences, Beijing \\ 100085, China \\ ${ }^{\mathrm{b}}$ Key Laboratory of Ion Beam Bioengineering, Hefei Institutes of Physical Science, Chinese Academy of Sciences and Anhui Province, Hefei, Anhui 230031, \\ China \\ ${ }^{\mathrm{c}}$ Department of Urology, The Second Hospital of Tianjin Medical University, Tianjin Institute of Urology, Tianjin 300211, China \\ d Institute for Medical Engineering and Science, Massachusetts Institute of Technology, Cambridge, Massachusetts, USA
}

\section{A R T I C L E I N F O}

\section{Article history:}

Received 22 November 2015

Received in revised form

23 December 2015

Accepted 9 January 2016

Available online 12 January 2016

\section{Keywords:}

miR-214

Nrf2

ATF4

EZH2

Erythroid cells

\begin{abstract}
A B S T R A C T
Nuclear factor (erythroid-derived 2) like 2 (Nrf2) is a key regulator in protecting cells against stress by targeting many anti-stress response genes. Recent evidence also reveals that Nrf2 functions partially by targeting mircroRNAs (miRNAs). However, the understanding of Nrf2-mediated cytoprotection through miRNA-dependent mechanisms is largely unknown. In the current study, we identified a direct Nrf2 targeting miRNA, miR-214, and demonstrated a protective role of miR-214 in erythroid cells against oxidative stresses generated by radiation, excess iron and arsenic (As) exposure. miR-214 expression was transcriptionally repressed by Nrf2 through a canonical antioxidant response element (ARE) within its promoter region, and this repression is ROS-dependence. The suppression of miR-214 by Nrf2 could antagonize oxidative stress-induced cell death in erythroid cells by two ways. First, miR-214 directly targeted ATF4, a crucial transcriptional factor involved in anti-stress responses, down regulation of miR214 releases the repression of ATF4 translation and leads to increased ATF4 protein content. Second, miR214 was able to prevent cell death by targeting EZH2, the catalytic core component of PRC2 complex that is responsible for tri-methylation reaction at lysine 27 (K27) of histone 3 (H3) (H3K27me3), by which Asinduced miR-214 reduction resulted in an increased global H3K27me3 level and a compromised overexpression of a pro-apoptotic gene Bim. These two pathways downstream of miR-214 synergistically cooperated to antagonize erythroid cell death upon oxidative stress. Our combined data revealed a protective role of miR-214 signaling in erythroid cells against oxidative stress, and also shed new light on Nrf2-mediated cytoprotective machinery.
\end{abstract}

(c) 2016 Elsevier Inc. All rights reserved.

\section{Introduction}

Mammalian cells have evolved intrinsic defense mechanisms to antagonize exotic and intracellular stresses. For example, nuclear factor (erythroid-derived 2) like 2 ( Nrf2) serves as a key regulator for cellular protection against various damages in nearly all types of cells [1,2]. A considerable number of studies have demonstrated that Nrf2 signaling protects cells from many hazardous stimuli, such as radiation, invading pathogens and foreign chemicals (e.g.

Abbreviations: ATF-4, activating transcription factor 4; Bim, BCL2-like 11; EZH2, enhancer of zeste 2 polycomb repressive complex 2 subunit; eIF2, eukaryotic initiation factor-2; Fe-NTA, ferric nitrilotriacetate; Hri, eukaryotic translation initiation factor 2 alpha kinase 1; Nrf2, nuclear factor erythroid 2-related factor 2; ROS, reactive oxygen species

* Corresponding author at: 18 Shuangqing Road, Haidian, Beijing 100085, China.

E-mail address: sjliu@rcees.ac.cn (S. Liu). drugs through therapeutic interventions and environmental toxicants) [3-5]. Nrf2 directly targets a battery of roughly 250 genes responsible for a wide array of cellular antioxidant responses and detoxification processes, e.g. aldo-keto reductase, glutathione S-transferase, heme oxygenase-1, multidrug-resistance associated efflux pumps and $\mathrm{NAD}(\mathrm{P}) \mathrm{H}$ dehydrogenase quinone (NQO)-1 [6,7]. The level of Nrf2 protein is regulated by its inhibitor kelch-like ECH-associated protein (Keap1), and the binding of Keap1 to Nrf2 leads to its degradation in a ubiquitin-dependent manner $[7,8]$. Increased intracellular free radicals, such as reactive oxygen species (ROS), can trigger the dissociation of Keap1-Nrf2 complex, leading to nuclear translocation of Nrf2 and the transcriptional activation of its target genes through ARE in their promoter $[9,10]$. In fact, Keap1-Nrf2 complex is a most important cellular protection mechanism against a battery of toxic products including arsenic (As) [11-13], which could trigger oxidative stress and subsequently cause the modification changes of critical cysteine 
residues of Keap1 protein, resulting in the disruption of the contact between Keap1 and Nrf2 [11,14-16]. Meanwhile, burgeoning evidence suggests that Nrf2 also targets mircroRNAs (miRNAs) that are involved in cell metabolism, adhesion, tumorigenesis and so on $[17,18]$. However, whether Nrf2 also conducts cytoprotection through miRNA-dependent mechanisms is still largely unknown thus far.

During the maturation of proerythroblasts into red blood cells (RBCs), erythroid precursors experience various stresses from both inside and outside [19-22]. The increased demand of iron for hemoglobin synthesis in erythroblast results in enhanced formation of labile iron and consequently generation of free radicals, oxidative stress and cytotoxicity [19-21]. In addition, erythroid precursors may be also subjected to diverse stresses in bone marrow, such as hypoxia, mechanical stress, radiation, foreign chemicals and environmental toxicants [23-26]. Thus, an elegant network has evolved to protect erythroid cells during differentiation, including translation control to prevent hemoglobin aggregating by heme-regulated eIF2 $\alpha$ kinase (HRI), ATF4 signaling against oxidative stress and Nrf2 signaling-mediated protection, etc [22,27]. ATF4 is a key transcriptional factor involved in cytoprotection upon stresses, such as unfolded protein response, amino acids and oxidative stress, and ATF4 activation is a part of integrated stress response network $[28,29]$. For example, the ATF4 pathway is activated by HRI that phosphorylates eukaryotic translation initiation factor 2 , subunit $1 \alpha$, eIF $2 \alpha$ upon oxidative stress in erythroid cells, resulting in global inhibition of protein translation but selective enhanced translation of ATF4 through additional open reading frames (5'-ORF) in its 5'-untranslated region (5'-UTR) [22]. This is the primary mechanism underlying the regulation of ATF4 in response to stresses; however, whether ATF4 expression is also subjected to other regulatory mechanisms (such as Nrf2 signaling) remains largely unknown. It has been shown that oxidized phospholipids were able to activate both ATF4 and Nrf2 pathways, and Nrf2 acted as an upstream regulator of ATF4, although detailed molecular mechanisms were lacking [30].

Accumulating evidences suggested that miRNAs contribute to regulating erythropoiesis, and a few miRNAs are involved in erythropoiesis including proliferation of erythroid precursors, fetal $\gamma$ globin expression and enucleation [31-34]. It was also reported that miR-451 targeted 14-3-3zeta to activate FoxO3 pathway to protect erythroid cells from oxidant stress [35]. However, no miRNA has been described to affect survival of erythroid cells in response to external stresses in Nrf2-dependent manner. Moreover, although extensive investigations have been independently performed on the stress signaling pathways including Nrf2 and ATF4 signalings, detail crosstalk and links for them are not explicitly understood. In the current study, we aimed to exploit the potential contributions of miRNAs to the cytoprotection network in erythroid cells under external stress conditions. miR-214 has been demonstrated to modulate cell survival and apoptosis in other types of cells including cancer cells by directly targeting important cell survival-relevant genes [36,37]. A recent study also demonstrated that miR-214 directly targets ATF4 to restrain bone formation in osteoclasts by suppressing osteoclast activity [38]. Taken together, we hypothesized that miR-214 might be a part of the cellular defense system in protecting erthyroid cells against oxidative stress-induced toxicity. Our study verified our hypothesis and revealed a protective role of miR-214 in erythroid cells against oxidative stress.

\section{Materials and methods}

\subsection{Animal experimentation}

Male C57BL/6 mice of 6-8 weeks old and pregnant ICR mice were purchased from the Vital River Laboratories (Beijing, China). Mice were housed in temperature and humidity controlled animal facility with a 12-h light/dark cycle, and had free access to water and food. Experimental procedures were approved by the Animal Ethics Committee at the Research Center for Eco-environmental Sciences, Chinese Academy of Sciences.

\subsection{Cell culture and treatment}

Mouse erythroleukemia (MEL) cells were obtained from the Cell Resource Center of the Institute of Basic Medical Sciences (CAMS, China), and cultured in RPMI 1640 medium (Hyclone, CA USA) supplemented with $10 \%$ bovine calf serum (Hyclone). Cells were treated with various reagents including tertiary butylhydroquinone (TBHQ) and ferric nitrilotriacetate (Fe-NTA) (SigmaAldrich, St. Louis, MO, USA). Cell death was determined with a kit from BD Biosciences (San Diego, CA, USA), according to an established method [24].

\subsection{Plasmid construction and small RNA molecule transfection}

FLAG-Keap1, pGL3/Basic and PGL3/ARE expression plasmids were kindly provided by Prof. Xiaoming Yang in Beijing Institute of Radiation Medicine, Beijing, China. EZH2-3'UTR, EZH2-mutant 3 UTR, ATF4-3'UTR, ATF4-mutant 3'UTR, miR-214+ARE and miR$214 \triangle$ ARE were constructed into the luciferase reporter vector PGL3 vector. Mouse Nrf2, EZH2, Bim and miR-214 shRNA constructs were cloned into lentiviral vector PLKO.1, respectively. Lentiviral particle preparation and stable shRNA-based knockdown cell lines were performed, as described previously [39]. The primer sequences for vector construction are presented in Supplemental Table 1. The agomir RNA oligos of miR-214 and scrambled control molecules were purchased from GenePharma Biotechnology (Shanghai, China). With respect to small RNA molecule transfection, $20 \mu \mathrm{m} / \mathrm{ml}$ RNA molecules were transfected into cells using Lipofectamine 2000 (Invitrogen) according to manufacturer's instructions.

\subsection{Western blotting analysis}

Cells were harvested and then lysed in RIPA lysis buffer after washing with cold phosphate-buffered saline (PBS) (Solarbio, China) supplemented with protease inhibitor cocktail (Roche). Western blotting analysis was performed, as described previously [40]. Antibodies (Abs) against ATF4, Nrf2, GAPDH and FLAG were obtained from Santa Cruz Biotechnology (Santa Cruz, CA, USA). Abs against EZH2 and Bim were purchased from Cell Signaling Technology (Beverly, MA, USA). Ab against H3K27me3 was purchased from Millipore (Billerica, MA, USA). Abs against CD71 and Ter119 were purchased from eBioscence (San Diego, CA, USA). The intensity of autoradiogram in Western blot bands was calculated using software Image J (NIH, USA), the band intensity was normalized to that of according loading control.

\subsection{Luciferase reporter activity assay}

Luciferase reporter activity was carried out with the Dual-Luciferase Reporter Assay System (Promega, Medison, MI). Briefly, cells were transfected with luciferase reporter constructs for $24 \mathrm{~h}$ before cellular treatment, and were then harvested and incubated in $1 \times$ passive lysis buffer for $30 \mathrm{~min}$ at $4^{\circ} \mathrm{C}$. Finally, the luciferase 
activity was determined on a multifunctional microplate reader (Thermo Fisher Scientific).

\section{6. $q P C R$ analysis}

RNA extraction was performed using TRIzol reagent, and cDNA was synthesized using first strand cDNA synthesis kit according to the manufacturer's protocol (Thermo Fisher Scientific). mRNA expression levels of interest genes were measured using SYBR Green qPCR mix (Promega Inc., USA) on a Mx3005P qRT-PCR instrument (Bio-Rad). The primer sequences for real-time PCR are shown in Supplemental Table 1.

\subsection{Flow cytometry (FACS) analysis}

Cells were washed with cold PBS twice and then stained with different Abs for 30 min in dark at $4{ }^{\circ} \mathrm{C}$. Thereafter, FACS analysis was performed on a BD FACSCalibur ${ }^{\mathrm{TM}}$ (BD Biosciences), according to the established method as described previously [24].

\subsection{Chromatin Immunoprecipitation (ChIP) assay}

ChIP assay was done with a kit purchased from Upstate (Lake Placid, NY, USA). Briefly, cells were cross-linked with $1 \%$ formaldehyde at room temperature for $10 \mathrm{~min}$, and were then washed with cold PBS. Fixed cells were re-suspended in lysis buffer with SDS, followed by sonication of chromatin into fragment sizes ranging from 200 to $1000 \mathrm{bp}$ as previously described [40]. After sonication, the supernatant containing chromatin was diluted in ChIP dilution buffer, and was subjected to incubation with Abs against IgG, Nrf2 and H3K27me3. Target sequences from purified chromatin DNA were amplified and quantified by qPCR. Primer sequences for ChIP-PCR are shown in Supplemental Table 1.

\subsection{Statistical analysis}

All data are presented as means $\pm \mathrm{SD}$, and statistical analysis was determined by independent $t$ test or One-Way ANOVA test. Statistical significance was determined with $P<0.05$.

\section{Results}

\section{1. miR-214 expression is repressed in erythroid cells upon stress}

To investigate the possible role of miR-214 in protecting erythroid cells, we examined its expression during differentiation and upon diverse stresses. miR-214 levels were measured at different stages of erythroid differentiation in E14.5 fetal liver cells, MEL and K562 cells by qPCR. Progression of erythropoiesis in fetal liver was classified into distinct stages from S0 to S4, as characterized by S0: CD71 ${ }^{\text {low }}$ Ter119 ${ }^{\text {low }}$ S1: CD71 ${ }^{\text {hi }}$ Ter119 ${ }^{\text {low }}$, S2: CD71 ${ }^{\text {hi }}$ Ter119 ${ }^{\text {Mid }}$, S3: CD71 ${ }^{\text {hi }}$ Ter119 ${ }^{\text {high }}$, and S4: CD71 ${ }^{\text {high }}$ Ter119 ${ }^{\text {low }}$ (Fig. 1A), representing the progression from erythroid primitive progenitors to proerythroblasts, basophilic erythroblasts, chromatophilic erythroblasts, orthochromatophilic erythroblasts and finally reticulocytes and erythroblasts. As shown in Fig. 1A, the expression level of GATA-1 was dramatically upregulated during differentiation from S0 to S3 $(P<0.05)$. Additionally, the mRNA expression of $\beta$-globin was also markedly increased upon erythroid differentiation (Supplementary Fig. 1). In contrast, miR-214 expression showed a significant reduction from S0 stage to $\mathrm{S} 1, \mathrm{~S} 2$ and S3 (Fig. $1 \mathrm{~B}, P<0.05$ ).
A

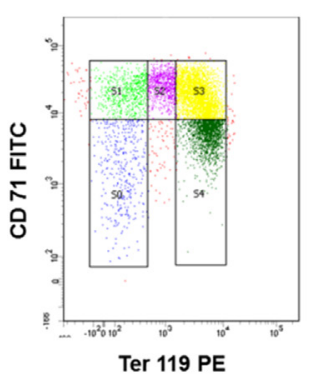

D

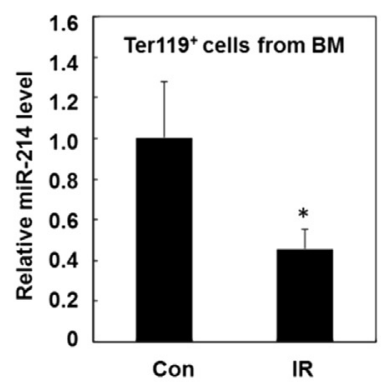

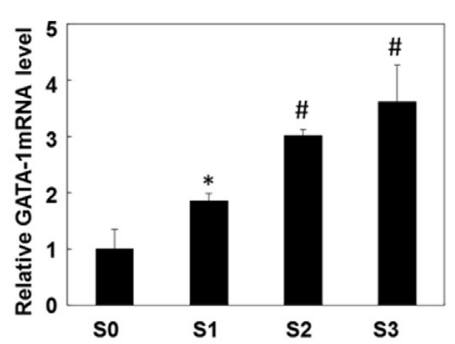

$\mathbf{E}$

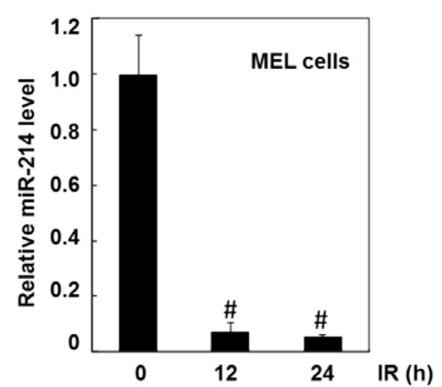

B

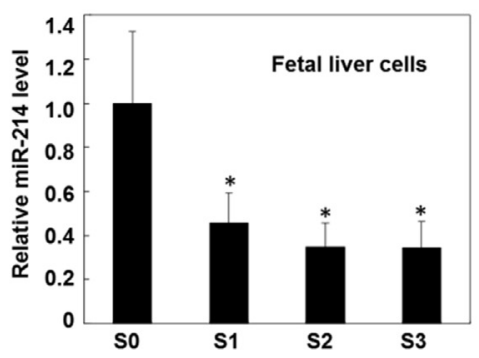

$\mathbf{F}$

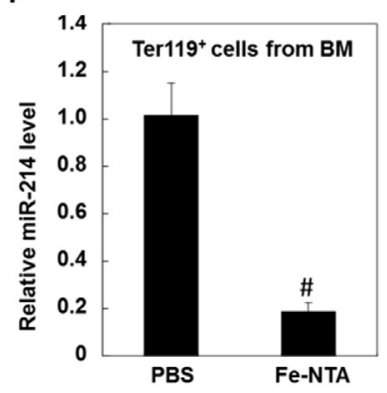

C

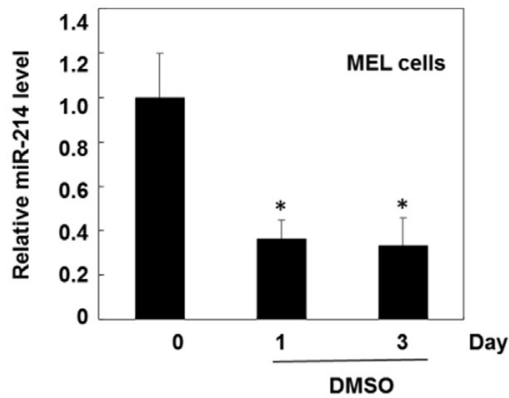

G

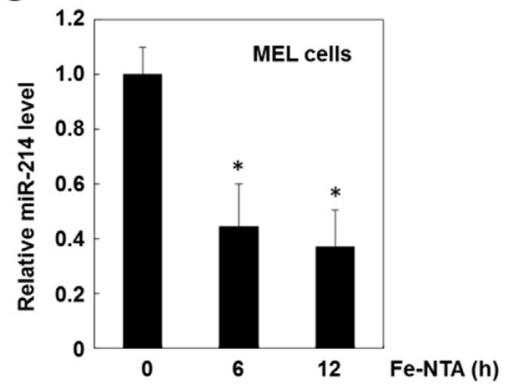

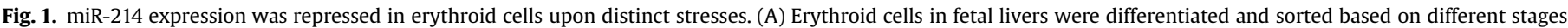

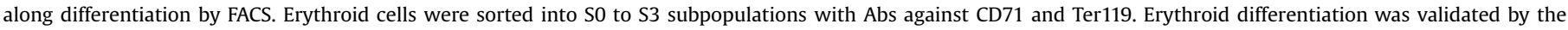

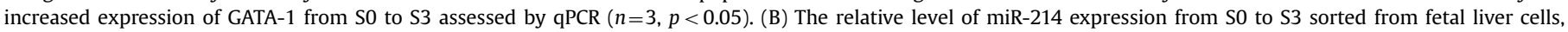

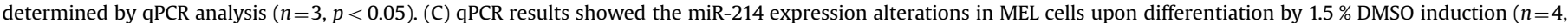

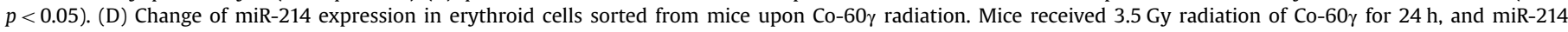

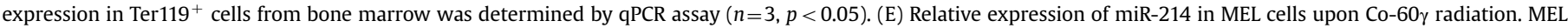

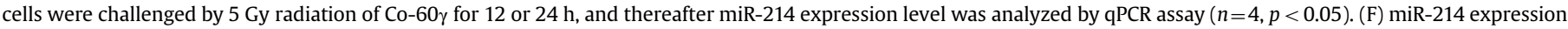

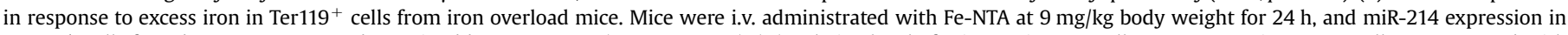

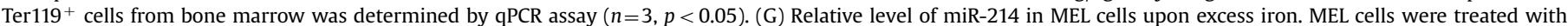
$100 \mu \mathrm{M}$ Fe-NTA for 6 or $12 \mathrm{~h}$, followed by qPCR analysis $(n=4, p<0.05)$. 
Afterwards, miR-214 expression was also assessed in differentiated MEL cells induced by DMSO and differentiated K562 cells induced by hemin, respectively. Similar to the results from fetal liver cells, the expression level of miR-214 was considerably hampered in both MEL cells and K562 cells upon differentiation (Fig. 1C and Supplementary Fig. 2, $P<0.05$ ). These data indicated restrained expression of miR-214 during erythroid differentiation.

To explore the biological significance of miR-214 reduction in stress-induced cytotoxicity, erythroid cells were exposed to various stress stimuli. Previous studies demonstrated that ionizing radiation induces substantial oxidative stress and severe damage in erythroid cells $[41,42]$. Thus, we determined the expression of miR-214 in erythroid cells challenged by Co-60 $\gamma$ radiation at sublethal dosages in vivo and in vitro. As shown in Fig. 1D, the expression level of miR-214 was reduced by more than 50 \% in BM Ter119 ${ }^{+}$erythroid cells from mice post radiation, relative to untreated control $(P<0.05)$. Similarly, miR-214 expression was also markedly compromised by over $90 \%$ in MEL cells upon exposure to $5 \mathrm{~Gy}$ Co-60 $\gamma$ radiation for 12 or $24 \mathrm{~h}(P<0.05)$ (Fig. $1 \mathrm{E})$. In addition, excess iron could also cause oxidative stress, resulting in detrimental effects on survival of erythroid cells $[43,44]$. Therefore, alterations of miR-214 expression were also determined in mice and MEL cells upon excess iron stimulus. As shown in Fig. 1F, miR-214 level decreased more than $80 \%$ in BM Ter119+ erythroid cells from Fe-NTA treated mice, compared to that in untreated mice $(P<0.05)$. The expression level of miR-214 in MEL cells was also reduced by over $50 \%$ in response to Fe-NTA treatment, compared to that in untreated cells $(P<0.05)$.

\subsection{ROS-dependent downregulation of miR-214 in erythroid cells}

A number of previous studies demonstrated that the expression changes for certain miRNAs could be attributed to increased ROS production [45-48]. To elucidate the mechanism responsible for miR-214 down regulation in erythroid cells in response to stress, we investigated the relationship between miR-214 expression and ROS production. In agreement with previous studies
[49,50], Co-60 $\gamma$ radiation and Fe-NTA gave rise to ROS generation by 2-3 folds, compared to untreated control (Fig. 2A and C), whereas the expression of miR-214 decreased significantly (Fig. 1E and G). This observation suggested that ROS generation reversely correlated to miR-214 expression. To assess the contribution of ROS to the regulation of miR-214 expression, a potent ROS scavenger, NAC, was used to quench intracellular ROS (Supplementary Fig. $3, P<0.001)$. NAC pre-treatment greatly reversed the reduction of miR-214 expression upon radiation and excess iron by approximately 7 folds and 4 folds, respectively, compared to the cells without NAC pre-treatment (Fig. $2 \mathrm{~B}$ and D, $P<0.001$ ). Analogous to previous studies [51,52], intracellular ROS generation in MEL cells was significantly increased by $50 \%$ upon erythroid differentiation induced by DMSO $(P<0.05)$, and concurrent $60 \%$ reduction of miR-214 expression was observed (Figs. 1C and 2E). Reduction of intracellular ROS by NAC pre-treatment considerably promoted 4-fold increase of miR-214 level, compared to induced cells without NAC pre-treatment (Fig. $2 \mathrm{~F}, P<0.001$ ). Furthermore, similar results were obtained in cells upon As exposure (Fig. 2G and $\mathrm{H}$ ). Taken together, these data suggested that repression of miR-214 expression were partially dependent on ROS generation under stresses.

\subsection{Nrf2 transcriptionally drives miR-214 expression}

Since Nrf2 plays a critical role in anti-oxidant stress response, a possible regulation of the transcription of miR-214 by Nrf2 was examined. Based on the bioinformatic analysis, a conserved ARE was found at -855 to $-846 \mathrm{bp}$ within the promoter region of miR-199a/214 cluster (Fig. 3A). The canonical ARE typically harbors a consensus sequence of $5^{\prime}$ TGAC-nnn-GC-3' that could be specifically recognized by Nrf2, and the binding to ARE by Nrf2 is fundamentally involved in anti-oxidative stress responses $[3,53]$. To interrogate the binding of Nrf2 to the ARE within its promoter region, the ChIP assay in MEL cells was performed. As shown in Fig. 3A, significant enrichment was verified within the promoter of miR-214 upon Nrf2 Ab precipitation, demonstrating the binding of
A
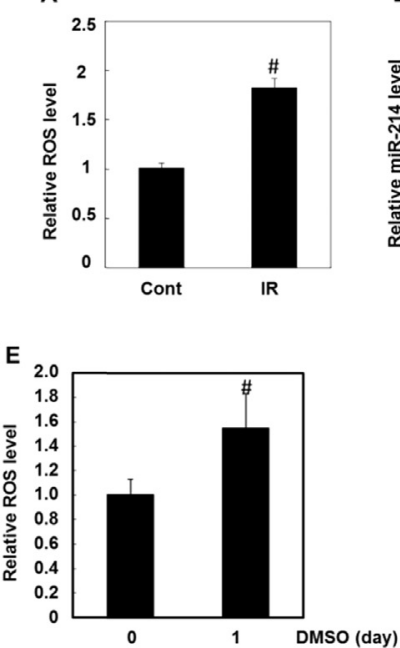

B
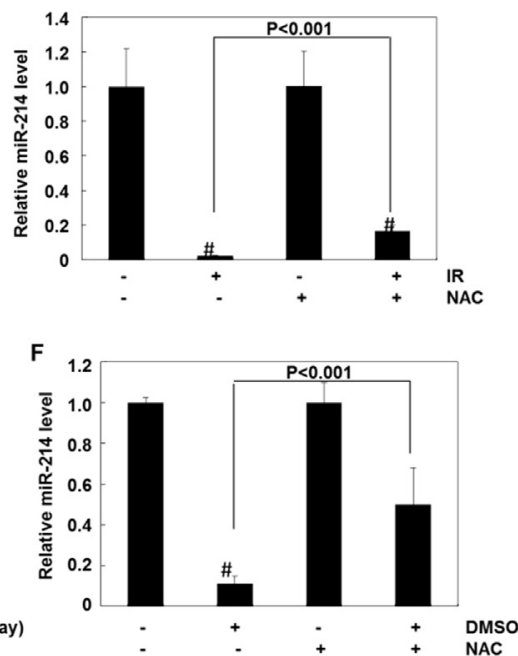

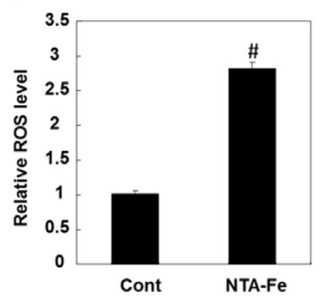

G

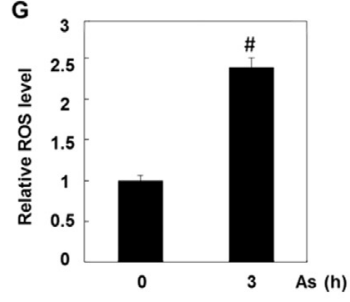

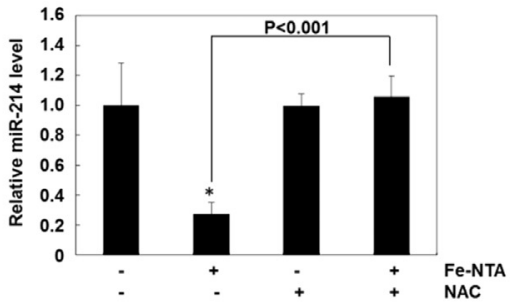

$\mathrm{H}$

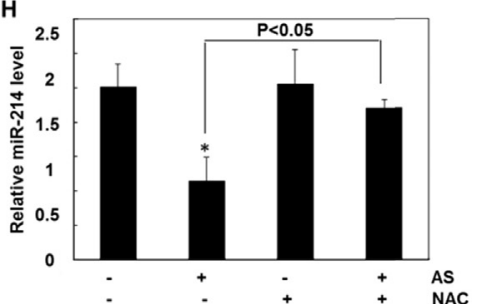

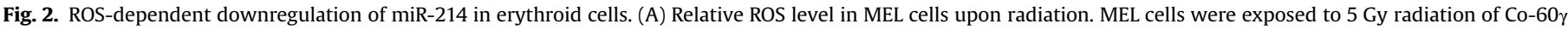

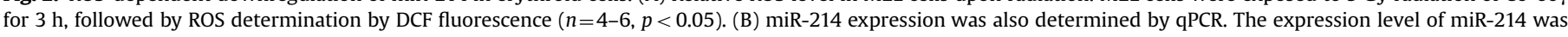

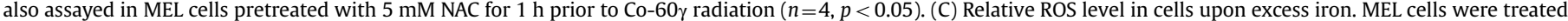

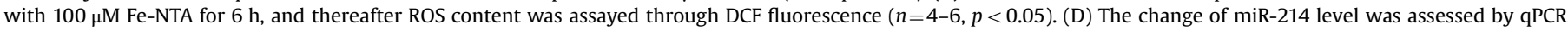

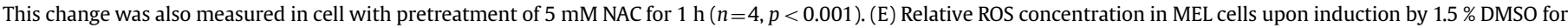

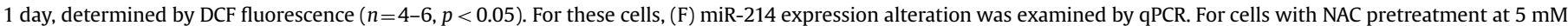

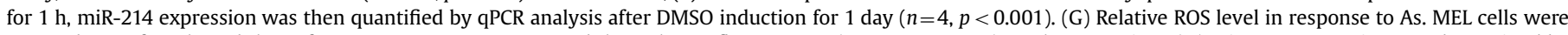

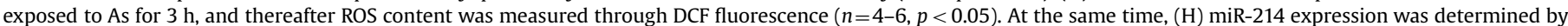
qPCR assay. Meanwhile, miR-214 level was assessed by qPCR assay in MEL cells with pretreatment of $5 \mathrm{mM}$ NAC for $1 \mathrm{~h}$ prior to As exposure ( $n=4, p<0.05$ ). 

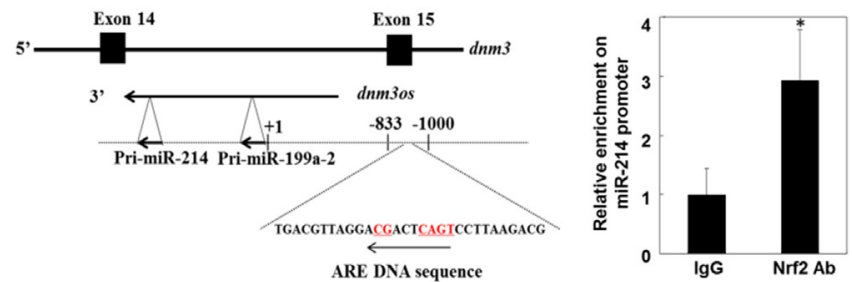

D
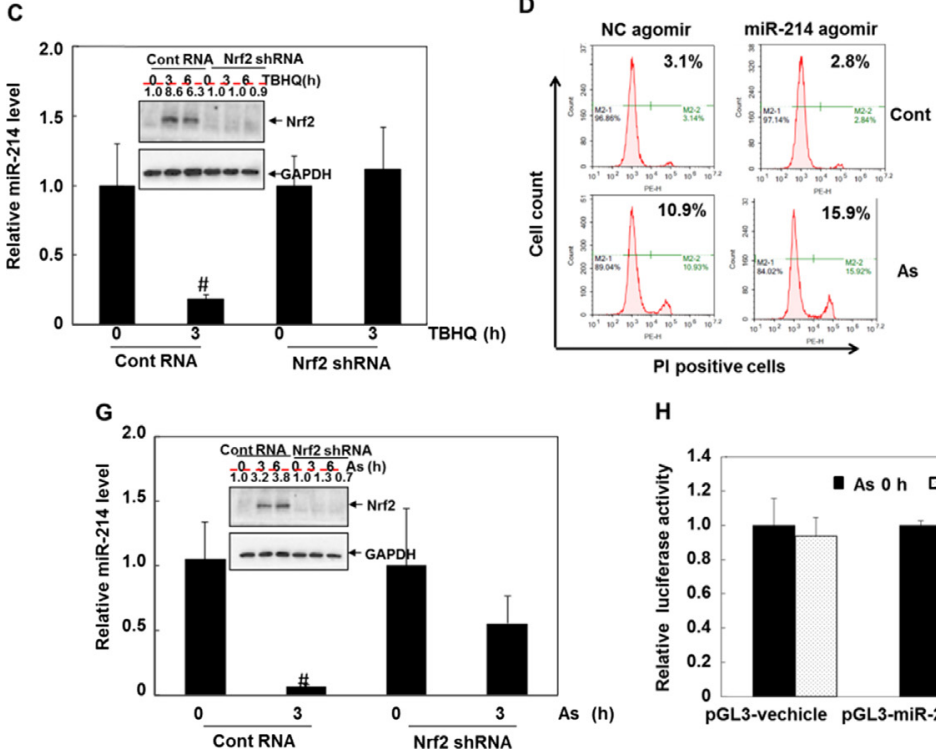

B
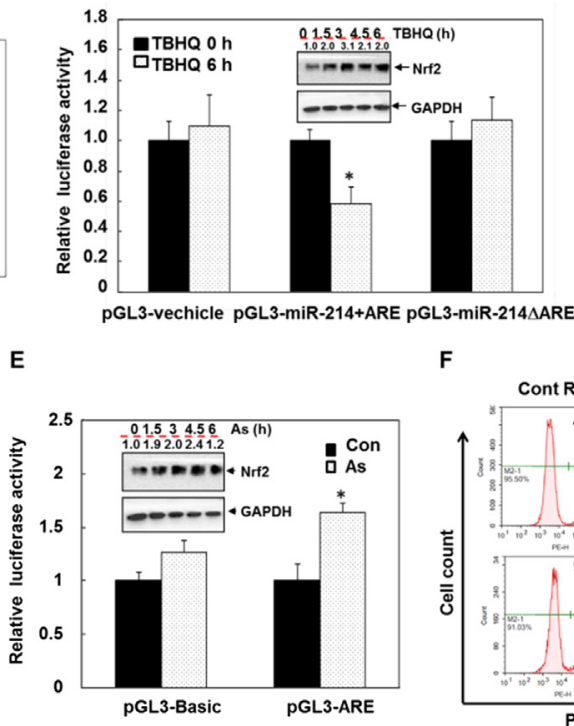

H

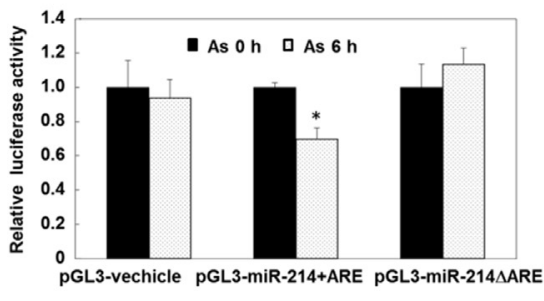

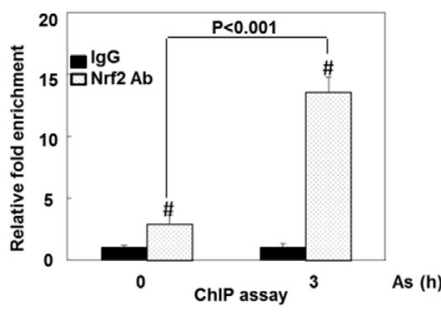

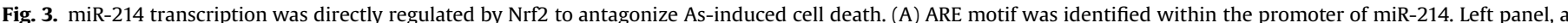

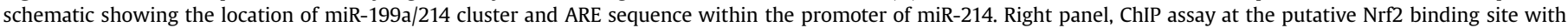

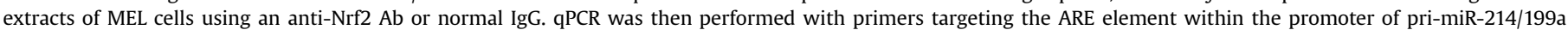

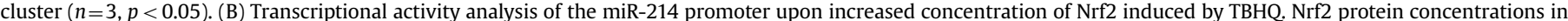

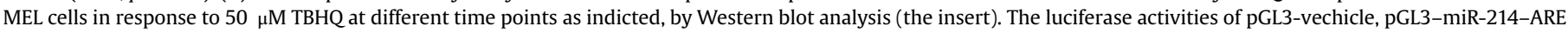

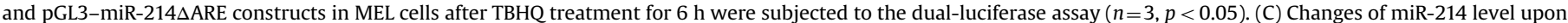

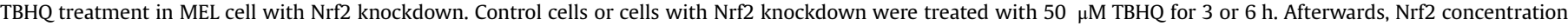

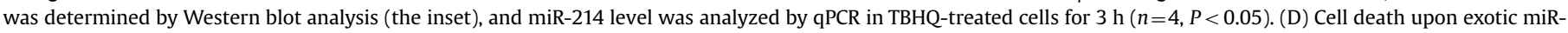

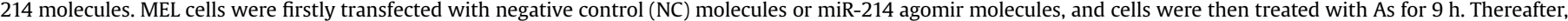

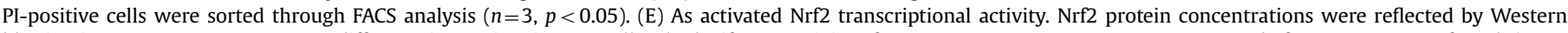

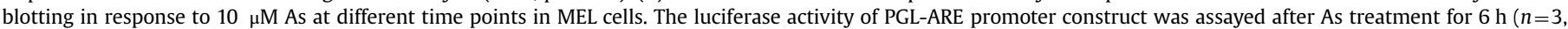

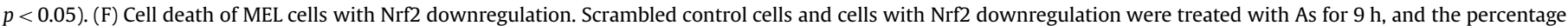

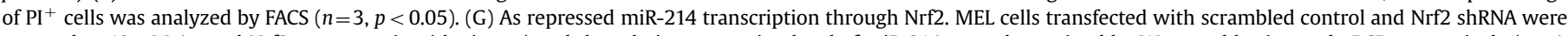

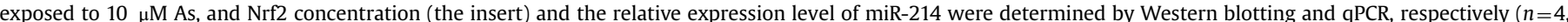

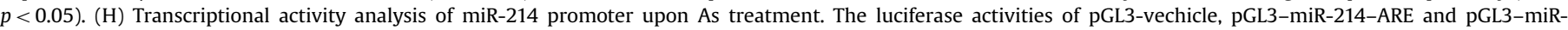

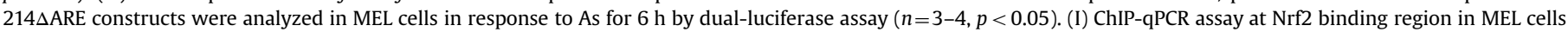
post As treatment for $3 \mathrm{~h}$ using an anti-Nrf2 Ab or normal $\operatorname{IgG}(n=3-4, p<0.001)$.

Nrf2 to the promoter of miR-214. This finding also implied direct transcription of miR-214 by Nrf2. To test this hypothesis, TBHQ, a selective Nrf2 activator, was used to instigate Nrf2 transcriptional activity. As shown in Fig. 3B (the insert blotting autoradiogram), TBHQ robustly increased the protein level of Nrf2 $6 \mathrm{~h}$ post-treatment in MEL cells. In addition, TBHQ treatment significantly suppressed the relative luciferase activity of pGL3-miR-214-ARE which contains the miR-214 promoter with intact ARE sequence by $41 \%$, compared to the untreated control (Fig. $3 \mathrm{~B}, P<0.05$ ). However, this suppression was not observed for pGL3-miR$214 \triangle \mathrm{ARE}$ (namely with deletion of the ARE sequence within the miR-214 promoter) (Fig. 3B). To substantiate this observation, additional experiments were carried out. Nrf2-knockdown MEL cells were screened, where endogenous Nrf2 was stably diminished through lentiviral shRNA constructs. As shown in the insert in Fig. 3C, TBHQ-induction of Nrf2 was compromised upon Nrf2 shRNA lentiviral infection. Relative to scrambled control, the reduction of miR-214 expression was negligible in Nrf2-knockdown MEL cells (Fig. 3C), indicating the vital role of Nrf2 in miR-214 expression.

To explore the biological impact of miR-214 down-regulation upon oxidative stress in erythroid cells, MEL cells were treated with arsenic. As shown in Fig. 3D, As induced ca. 8\% increase of cell death in MEL cells, compared to untreated control $(P<0.05,3 \%$ vs $11 \%)$, similar to previous studies [22]. Importantly, cell death was augmented by approximately $50 \%$ in cells transfected with miR214 agomir molecules, relative to scrambled molecule-transfected cells (Fig. 3D, $16 \%$ vs $11 \%, P<0.05$ ). Reversely, cell death was also significantly restrained by $>50 \%$ for MEL cells where endogenous miR-214 expression was selectively repressed through shRNA knockdown (Supplementary Fig. 4, 10.4\% vs $4.6 \%, P<0.05$ ). These results demonstrated that repression of miR-214 was necessary to protect cells against As-induced cell death.

Nrf2 induction is critical against As stress [14,54]. Consistent with previous results [14], As treatment greatly induced Nrf2 protein content in MEL cells, and subsequentially increased the luciferase reporter activity of pGL3/ARE (an established construct to assay the transcriptional activity of Nrf2 [55]) in MEL cells 
(Fig. 3E, $P<0.05$ ). Furthermore, As-induced cell death was significantly promoted in MEL cells by Nrf2 knockdown (Supplementary Fig. 5 and Fig. 3F, 13\% vs 9\%, $P<0.05$ ). Meanwhile, Asinduced repression on miR-214 expression was nearly revered by a transcriptional inhibitor, actinomycin D, confirming the transcriptional regulation of miR-214 by Nrf2 upon As stress (Fig. S6).
The relationship between Nrf2 induction and miR-214 transcriptional repression in response to As was further investigated. As shown in Fig. 3G, As-induced miR-214 reduction was greatly blocked ( 8 folds) in cells with stable Nrf2 knockdown, in comparison to scrambled control $(P<0.05)$. In addition, As treatment diminished the luciferase activity of pGL3-miR-214-ARE but not

A

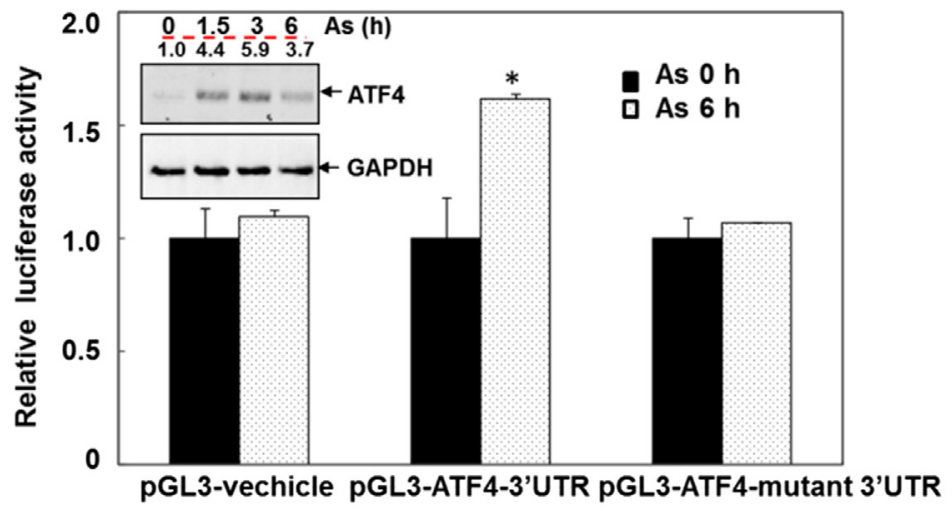

B

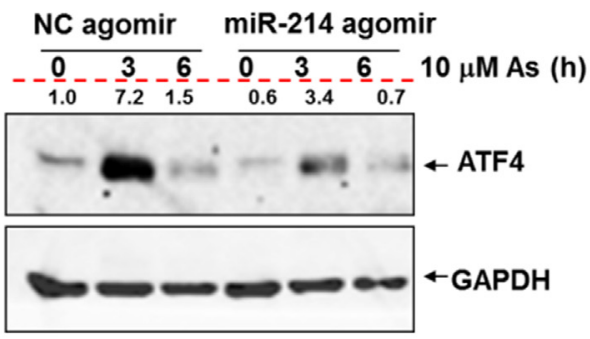

C

Cont RNA ATF4 shRNA

0

$1.0-4.34 .0-0.5-1.5-2.4$
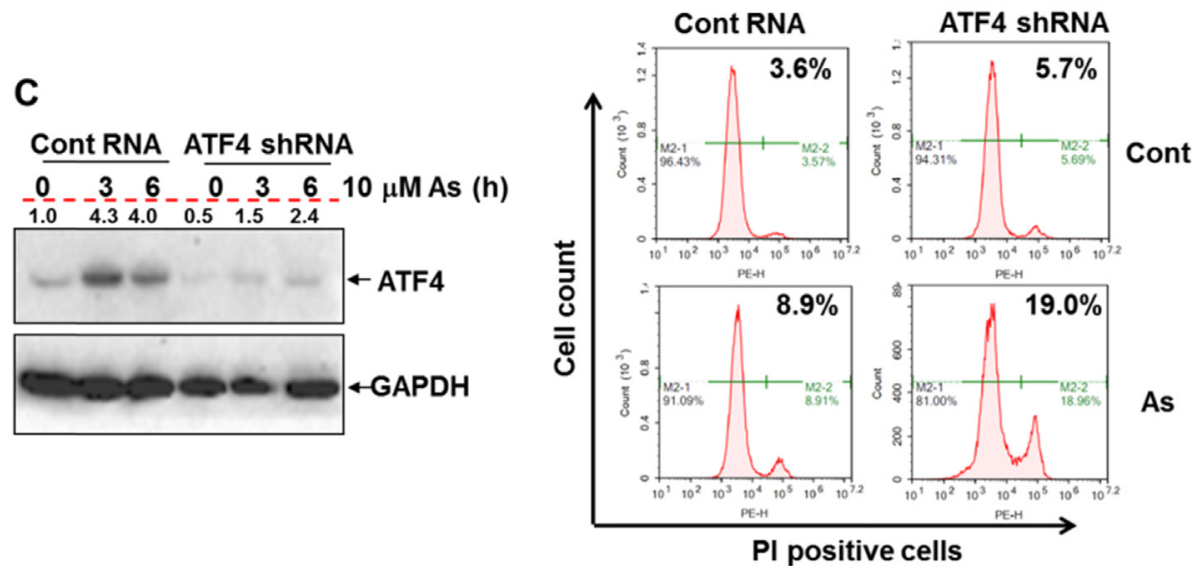

D

Cont RNA Nrf2 shRNA
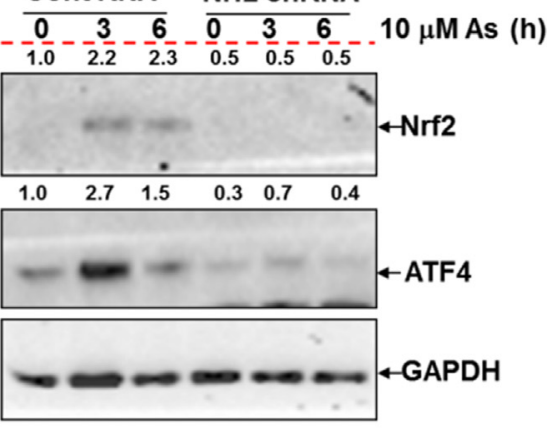

PI positive cells

E

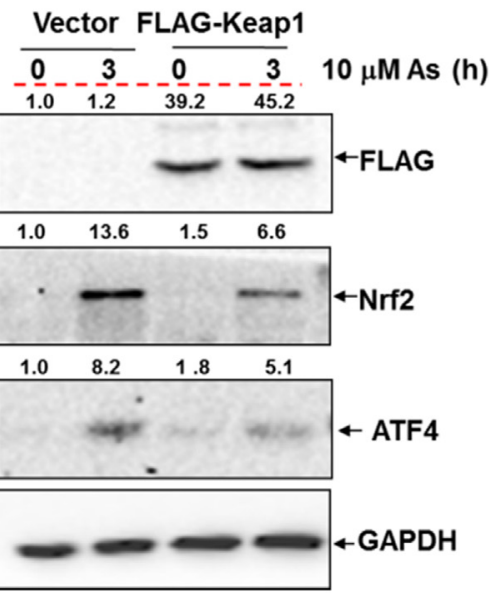

$\mathbf{F}$
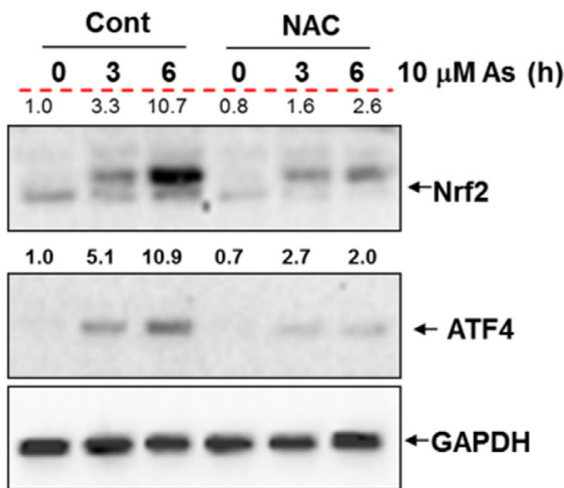

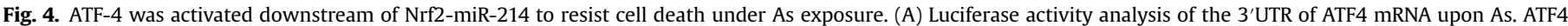

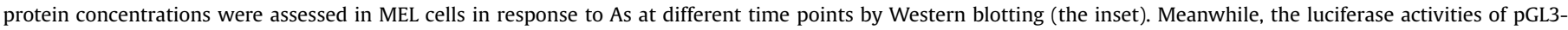

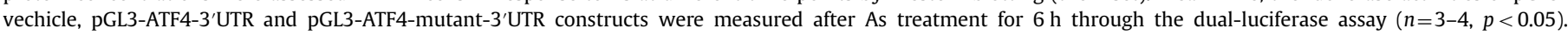

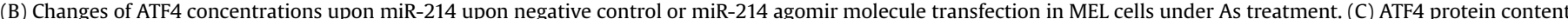

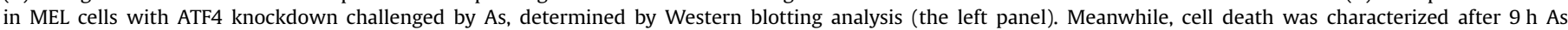

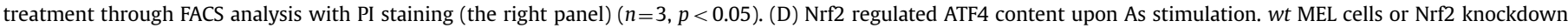

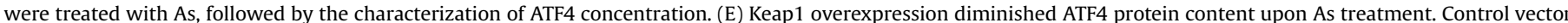

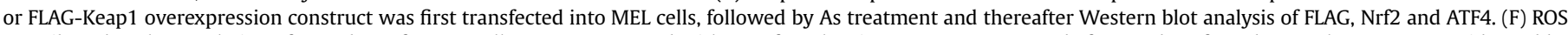

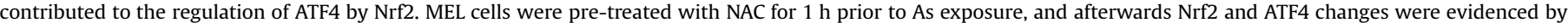
Western blot. 
A

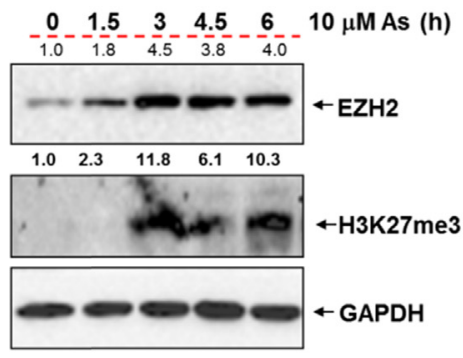

B

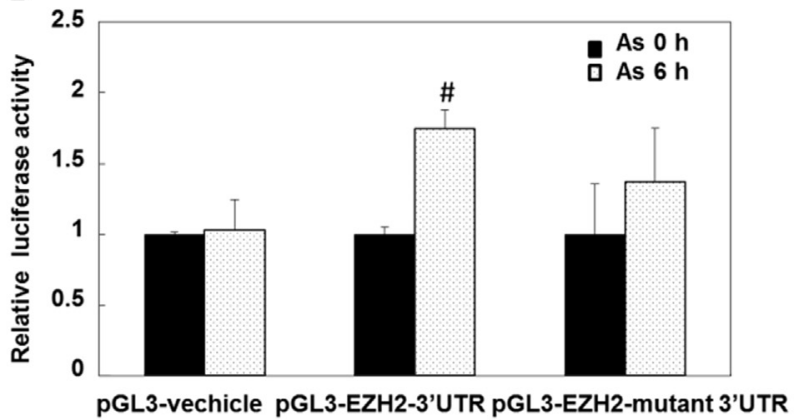

C

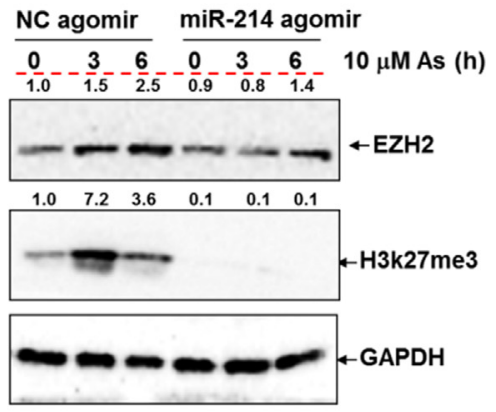

D
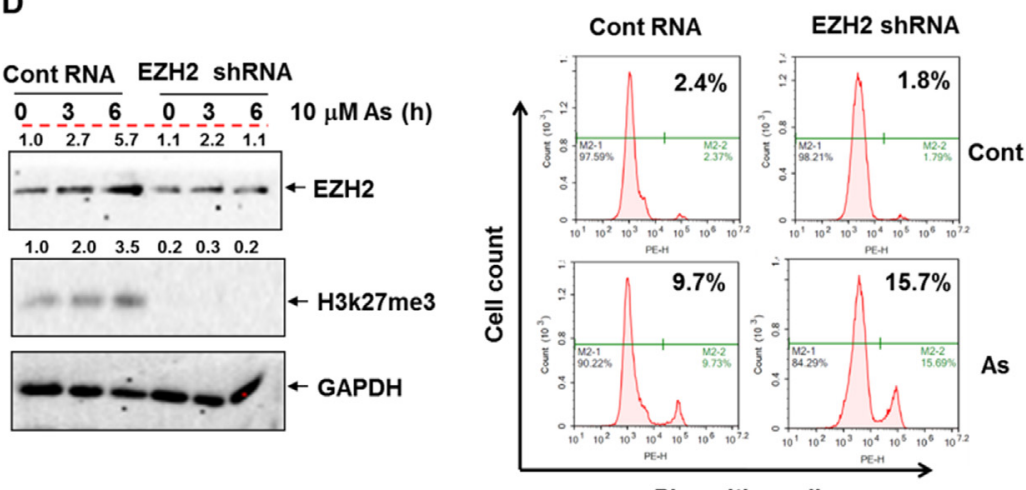

E

PI positive cells

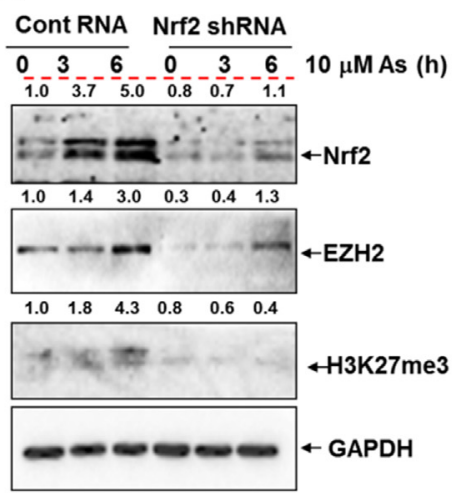

F

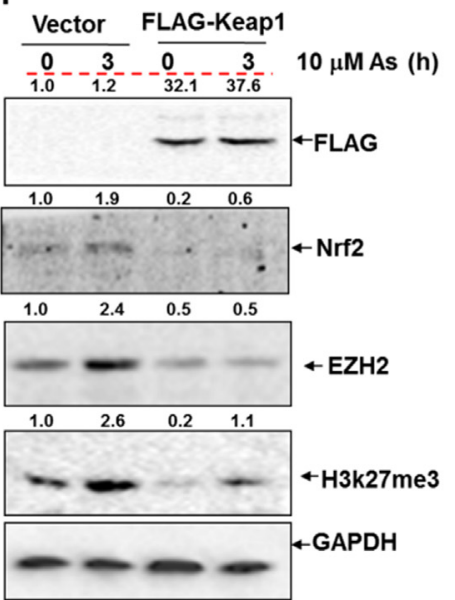

G

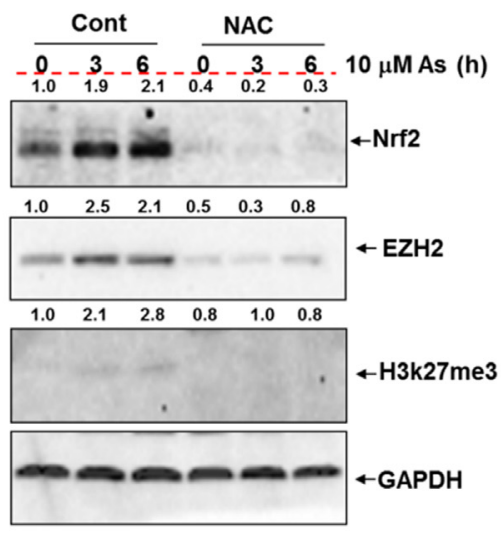

Fig. 5. miR-214 targeted EZH2 against As-induced cell death. (A) As treatment increased EZH2 and H3K27me3 levels. MEL cells were treated with As, and EZH2 content and global H3K27me3 levels were determined by Western blot analysis at different time points. (B) Luciferase activities of pGL3-vechicle, pGL3-EZH2-3'UTR and pGL3-EZH2mutant-3'UTR constructs were measured in MEL cells upon As exposure by the dual-luciferase assay $(n=3-4, P<0.05)$. (C) EZH2 content and global H3K27me3 level in MEL cells transfected with negative control molecules or miR-214 agomir molecules in response to As. (D) EZH2 regulated cell death. Control shRNA or EZH2 shRNA constructs were first stably transfected into MEL cells (the left panel). EZH2 concentration and global H3k27me3 level were determined in these cells treated with As for 3 or $6 \mathrm{~h}$. Meanwhile, cell death was also examined after $9 \mathrm{~h}$ As treatment through FCAS with PI staining $(n=3, P<0.05)$. (E) Nrf2 regulated EZH2 and H3K27me3 concentrations upon As stimulation. EZH2 and H3K27me3 concentrations were assessed in MEL cells with or without Nrf2 knockdown challenged by As exposure. (F) Keap1 overexpression compromised EZH2 and global H3K27me3 contents. The levels of Nrf2, EZH2 and global H3k27me3 modification were analyzed in MEL cells with FLAG-Keap1transfection in response to As treatment, determined by Western blotting. (G) ROS modulated the levels of EZH2 and global H3k27me3 modification. MEL cells were firstly pre-treated with NAC for $1 \mathrm{~h}$ before As exposure, and afterwards Nrf2, EZH2 and H3K27me3 levels were determined by Western blot analysis.

pGL3-miR-214 $\triangle$ ARE by $30 \%$, compared to the untreated cells $(P<0.05)$ (Fig. $3 \mathrm{H})$. ChIP assays also revealed more than 4 -fold $\mathrm{Nrf} 2$ enrichment in miR-214 promoter region in As-treated cells, compared to untreated cells (Fig. $3 \mathrm{I}, P<0.001$ ), substantiating the transcriptional regulation of miR-214 by Nrf2 upon As treatment. Collectively, these data demonstrated the modulation of miR-214 expression by Nrf2 at the transcription level, and also identified the crucial role of Nrf2-miR-214 signaling in protecting cells against As-induced cytotoxicity.

\subsection{Nrf2-miR-214 targets ATF4 to protect cells against As toxicity}

To delineate the molecular mechanisms underlying Nrf2-miR214 signaling in protecting erythroid cells, we surveyed the downstream targets of miR-214. A recent study demonstrated that miR-214 directly targets ATF4 to restrain bone formation by suppressing osteoclast activity [38]. ATF4 plays a fundamental role in maintaining metabolic homeostasis and cell survival, and its induction is crucial for cells against diverse stresses including endoplasmic reticulum (ER) stress, oxidative stress and amino acid 
depletion [56-58]. Therefore, we hypothesized that miR-214 may also directly target ATF4 to initiate the protective mechanism in erythroid cells in response to oxidative stress. To test the hypothesis, we examined the regulation of ATF4 by miR-214. As shown in Supplementary Fig. 7A, miR-214 agomir molecules reduced endogenous ATF4 protein level in MEL cells, and miR-214 agomir molecules also significantly suppressed the luciferase activity of pGL3/ATF4-3'UTR $(P<0.05)$, without suppression on the luciferase activity of pGL3/ATF4-mutant 3'UTR (Fig. S7B). In agreement with previous reports [22,24,25], As treatment greatly enhanced ATF4 protein level in MEL cells and ex vivo cultured fetal liver cells (Fig. 4A and Supplementary Fig. 8). The luciferase activity of pGL3/ATF4-3'UTR was elevated by more than $60 \%$ in MEL cells with As treatment $(P<0.05)$, whereas the luciferase activity of pGL3/ATF4-mutant 3'UTR remained unchanged (Fig. 4A). Meanwhile, the endogenous ATF4 mRNA level were not significantly changed (Supplementary Fig. 9). Furthermore, transfection of miR-214 agomir molecules remarkably diminished ATF4 protein level in As-treated MEL cells (Fig. 4B). All considered, these data support a direct regulation of ATF4 by miR-214 through a posttranscriptional mechanism at its $3^{\prime} \mathrm{UTR}$.

These results lead us to investigate a critical contribution of Nrf2-miR-214-ATF4 signaling to the overall protective mechanism against cytotoxicity. To prove this assumption, we further embarked on this signaling pathway. First, ATF4 stable knockdown cells were screened through lentiviral shRNA constructs (Fig. 4C). Consistent with previous observations [59], cell death was nearly doubled in ATF4 knockdown cells upon As treatment $(P<0.05)$, verifying the indispensable role of ATF4 against oxidative stress. Second, As-induced ATF4 protein expression was substantially inhibited in MEL cells with Nrf2 knockdown, compared to the scrambled control (Fig. 4D). Third, the induction of Nrf2 and ATF4 protein by As was diminished upon overexpression of Keap1, an inhibitor of Nrf2 (Fig. 4E). In contrast, removal of ROS by NAC greatly reduced both the Nrf2 and ATF4 protein levels upon As exposure (Fig. 4F). These results are consistent with previous studies showing ATF4 as a downstream target of Nrf2 [30].

\subsection{EZH2 is a downstream target of Nrf2-miR-214 against oxidative stress-induced cell death}

In addition to ATF4, we also looked for additional targets that may contribute to the overall Nrf2-miR-214-mediated protective mechanisms. A previous study suggested that EZH2 was a downstream target of miR-214, and aberrant expression of miR-214 was associated with compromised skeletal muscle cell differentiation [60]. EZH2 is the catalytic core component of PRC2 complex which conducts tri-methylation reaction at lysine 27 (K27) of histone 3 (H3), resulting in increased level of H3K27me3 [61,62]. Fine-tuned regulation of EZH2 is closely implicated in cell proliferation, differentiation, senescence and apoptosis under normal settings; however, deregulated EZH2 is associated with a number of disorders including various cancers [48,54]. To this end, we here tested the hypothesis that EZH2 could be a downstream target of Nrf2-miR-214 in erythroid cells against exotic stress. As shown in Fig. 5A, As greatly enforced the EZH2 protein level and the H3K27me3 level. The luciferase activity of pGL3-EZH2-3'UTR with intact miR-214 binding motif was elevated by approximately 1.7 folds in As-treated MEL cells, compared to untreated cells (Fig. 5B, $P<0.05$ ). However, this elevation of luciferase activity was not observed in cells transfected with pGL3-EZH2-3'UTR with mutant miR-214 binding motif (Fig. 5B), and no change of the mRNA level of EZH2 was demonstrated (Supplementary Fig. 10). To substantiate this finding, further studies demonstrated that miR214 agomir molecule transfection significantly reduced EZH2 protein level and consequentially the level of H3K27me3 in As- treated MEL cells (Fig. 5C). These results thus showed a direct regulation of EZH2 expression at the post-transcription level by miR-214.

To demonstrate the implication of EZH2 in regulating cell death upon stress in erythroid cells, As-triggered cell death was determined in cells where endogenous EZH2 was stably knocked down through shRNA (Fig. 5D). The percentage of cell death was increased by nearly 2 folds in EZH2-knockdown cells, compared to scrambled control cells (Fig. 5D), demonstrating an important role of EZH2 induction in protecting cells from As stress. To depict the regulation of Nrf2-miR-214 signaling on EZH2, the levels of EZH2 and H3K27me3 were assessed in Nrf2-knockdown cells. Consistent with the results with miR-214 agomir molecules (Fig. 5C), Nrf2 reduction (Fig. 5E) resulted in a great decline of the EZH2 protein and H3K27me3 levels in response to As. Similarly, forced expression of Keap1 led to a constant decline of EZH2 and H3K27me3 content (Fig. 5F). Furthermore, the evolvement of ROS in the Nrf2-miR-214-EZH2 signaling was also validated, as ROS removal by NAC pre-treatment greatly diminished the protein levels of Nrf2 and EZH2, and the methylation level of H3K27me3 as well (Fig. 5G). Together, our results demonstrated that Nrf2-miR214 modulated EZH2 expression to protect erythroid cells from As stress.

\subsection{Nrf2-miR-214-EZH2 targets Bim to impede cell death upon stress}

Previous results demonstrated that EZH2 repressed Bim (an important pro-apoptotic member in the Bcl-2 family [63]) to sustain the survival of chronic myeloid leukemia cells by enhancing H3K27me3 modification at the promoter of Bim gene [64]. To this end, we postulated a similar regulation of Bim in erythroid cells in response to As. To determine the role of Bim in promoting cell death under As exposure in erythroid cells, stable Bimknockdown MEL cells were prepared (Fig. 6A). Upon Bim knockdown, cell death was largely restrained by approximately $40 \%$ in response to As stimulus, compared to scrambled control (Fig. 6A, $P<0.05)$. This result demonstrated that Bim played an important role in the regulation of survival for erythroid cells upon stress. As shown in Fig. $6 \mathrm{~B}$ and $\mathrm{C}$, upon reduction of EZH2 protein content and H3K27me3 level by an inhibitor of EZH2, 3-Deazaneplanocin A (DZNep), the protein and mRNA expression levels of Bim were greatly up-regulated, with more than 6 folds increase for Bim mRNA level, compared to untreated cells $(P<0.001)$. Moreover, the enrichment of H3K27me3 at the promoter of Bim was demonstrated in As-treated cells, compared to untreated cells (Fig. 6D, $>2.5$ folds, $P<0.001$ ). To support the above results, increased Bim expression was observed in both EZH2-knockdown cells and Nrf2-knockdown cells as well (Fig. 6E and F). Collectively, these data demonstrated that Bim was targeted by Nrf2-miR-214EZH2 signaling to rescue As-treated erythroid cells from death. To examine whether there are synergistic effects between ATF4 and Bim on cell survival, we performed additional experiments to simultaneously knock down both ATF4 and Bim in MEL cells. As shown in Fig. S11, the percentage of cell death was similar in ATF4 and Bim double-knockdown cells, relative to scrambled control cells. Meanwhile, cell death in ATF4 single knockdown cells was increased by nearly 2 folds, compared to that in ATF4 and Bim double-knockdown cells in response to As. These results together demonstrated that ATF4 induction and Bim repression synergistically acted to regulate the survival of erythroid cells upon As stress.

\section{Discussion}

Nrf2 is necessary to protect cells from ROS-induced damage 

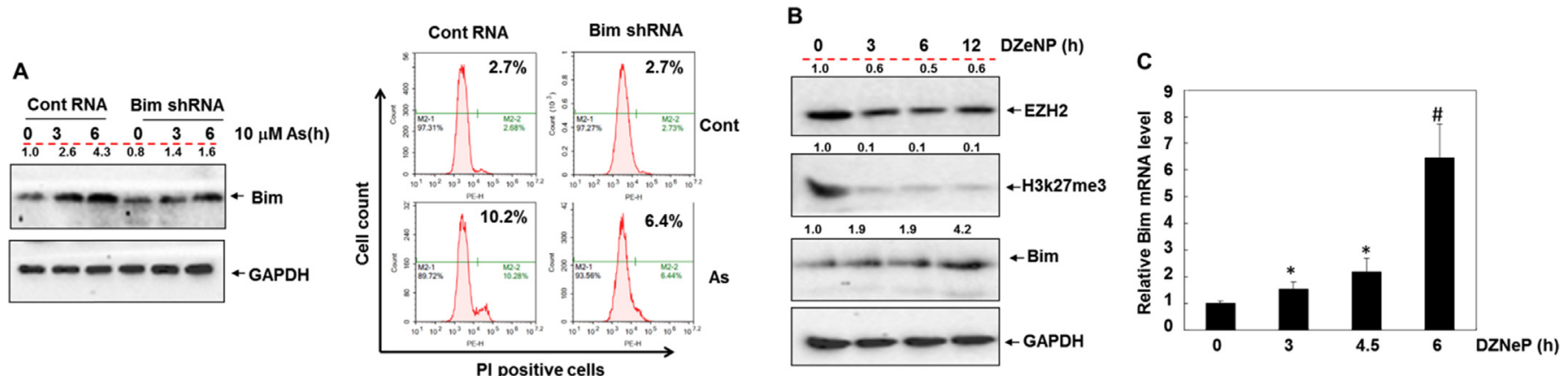

D

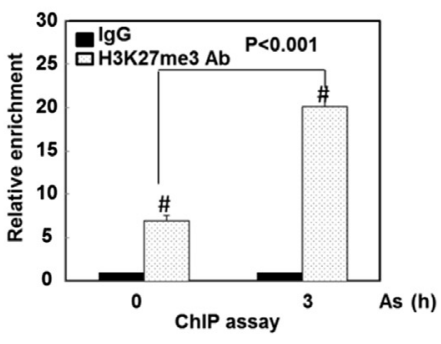

E
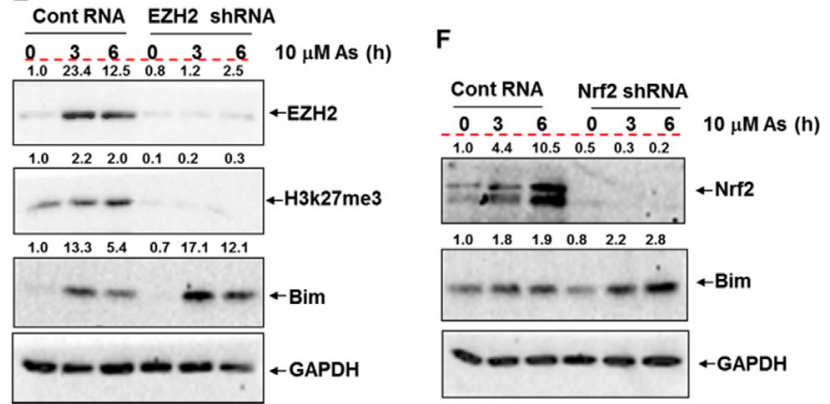

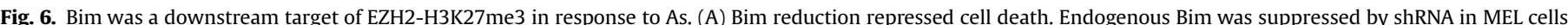

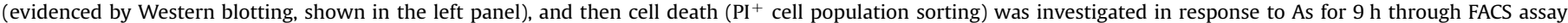

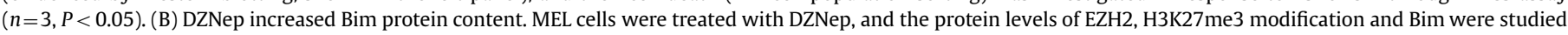

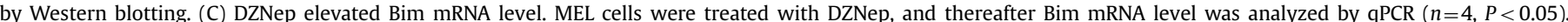

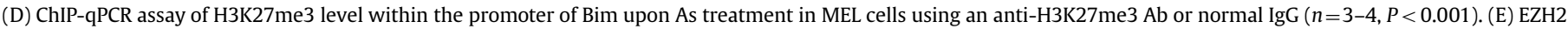

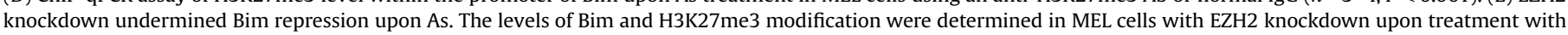

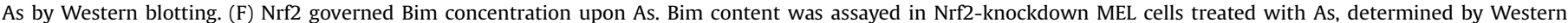
blotting.

upon endogenous and environmental stresses through modulating the expression of a myriad of cytoprotective and antioxidant genes $[1,2,7]$. In addition to protein-coding genes, a few miRNAs were recently identified to be directly regulated by Nrf2 to regulate cell metabolism and tumor progression $[17,18]$. Nonetheless, the implication of Nrf2-regulated miRNAs in anti-stress machinery is largely undefined. Mounting data demonstrate that miRNAs play important roles in directing erythropoiesis $[31,33]$. For instance, mice with deficiency of miR-144 and miR-451 showed such pronounced phenotypes as impaired late erythrocyte maturation, causing anemia and erythroid hyperplasia coupled to splenomegaly [65]. We here presented a novel function of miR-214 in protecting cells from oxidative stress-associated cell death through interlinking Nrf2 and ATF4, EZH2/Bim signaling in erythroid cells.

There is elevated formation of free radicals and increased oxidative stress in erythroid precursors due to enhanced demand of iron for hemoglobin synthesis and accumulated hemoglobin content $[20,66,67]$, and erythroid precursors may be also subjected to oxidative stress from foreign chemicals including drugs for medicinal purposes and environmental toxicants [19,22,68]. Therefore, erythroid cells are highly sensitive to oxidative stress, and aberrant ROS accumulation incurs impaired erythropoiesis, shortened lifespan of RBCs and even hemolysis [66,69,70]. A comprehensive protective machinery is employed in erythroid cells during differentiation. In this study, we identified a ROS-dependent manner of miR-214 repression upon oxidative stress from diverse treatments, and proved for the first time that this dependence was in parallel to the corresponding alterations of Nrf2 by ROS. Thus, this study highlighted the significance of Nrf2-miR-214 signaling (that is initiated by ROS) in promoting cell survival for erythroid cells upon exotic stresses.

Our recent studies demonstrated that ATF4 plays a crucial role against oxidative stress including As- and cadmium-induced impairments to erythroid cells [22,24]. The important role of Nrf2 in anti-stress responses has also been documented in erythroid cells $[14,71]$. Although evidence suggested that ATF4 could interact with Nrf2 to regulate the expression of antioxidant enzymes (e.g. heme oxygenase-l (HO-1)) [72], the crosstalk between these two cytoprotective pathways remains less understood. Previous mechanistic studies demonstrated that ATF4 is predominantly activated through eIF2 $\alpha$ phosphorylation by oxidative stress derived from unfolded proteins or As treatment, and its activation is necessary to antagonize cell death in erythroid cells upon these stresses $[22,70]$. In the current study, ATF4 was found to be activated by Nrf2 through repressing miR-214 expression. This finding thus added new insights into ATF4 activation mechanisms by Nrf2-driven miR-214 inhibition, and also signified an important contribution of ATF4 pathway to Nrf2-mediated cytoprotective machinery.

EZH2 is a catalytic core component of PRC2 complex and involved in numerous fundamental cellular processes, its dysregulation is closely involved in in tumor development and progression $[62,73]$. Though it was reported that PRC2 complex components including EZH2 was involved in epigenetic silencing of a subset of erythropoiesis-related genes $[74,75]$, the cytoprotective role of EZH2 in erythroid cells under stress conditions remains largely unknown thus far. Here, we uncovered a direct regulation of EZH2 by Nrf2 through miR-214 in response to oxidative stress, as As treatment increased EZH2 protein concentration by suppressing miR-214 level, associated with resultant elevation of global H3K27me3 level. As a result, increased H3K27me3 changes restrained the expression of pro-apoptotic gene Bim, through which oxidative stress-induced cell death was significantly reversed. Bim is a crucial downstream determinant in regulating cell viability and survival, and previous studies have demonstrated that Bim is a potent pro-apoptotic protein in initiating the intrinsic apoptotic 
pathway under both physiological and pathological conditions $[76,77]$. Bim level was also regulated by erythropoietin (EPO)mediated signaling in order to sustain the survival of erythroid cells $[78,79]$. Nevertheless, there is still no report on the implication of Bim alterations in regulating erythroid cells survival through miRNAs dependent manner to date. Therefore, our current study revealed the importance of miR-214-mediated Bim reduction in preventing cell death for erythroid cells against oxidative stress.

To summarize, we delineated a novel role of miR-214 in bridging Nrf2 and ATF4 signaling to provide antioxidant response by inhibiting Bim-induced pro-apoptotic effects through EZH2-dependent modification of histone methylation in erythroid cells. Despite the long established mechanisms underlying the cytoprotective role of $\mathrm{Nrf} 2$ in anti-stress responses, we here added new insights into Nrf2-mediated cytoprotective network: miR-214mediated regulation on ATF4 and EZH2/Bim. It should be noted that these two signaling pathways synergistically functioned to antagonize As-induced toxicity of erythroid cells. Our findings also pinpointed the Nrf2-miR-214 signaling as a novel therapeutic target for the treatment of oxidative stress-related erythroid disorders and hematological diseases.

\section{Competing interests}

There is no potential conflict of interests to disclose.

\section{Acknowledgments}

This work was supported by grants under the National "973" Program (Grant no.: 2014CB932000) and the Strategic Priority Research Program of the Chinese Academy of Sciences (Grant no. XDB14000000), grants from the National Natural Science Foundation of China (Grant nos.: 21507154, 21425731, 21377159, 21177151, 21207152). We thank all the laboratory members for their great assistance with experiments and reagents.

\section{Appendix A. Supplementary material}

Supplementary data associated with this article can be found in the online version at http://dx.doi.org/10.1016/j.freeradbiomed. 2016.01.005.

\section{Reference}

[1] Y. Huang, W. Li, Z.Y. Su, A.T. Kong, The complexity of the Nrf2 pathway: beyond the antioxidant response, J. Nutr. Biochem. 26 (12) (2015) 1401-1413.

[2] L.E. Tebay, H. Robertson, S.T. Durant, S.R. Vitale, T.M. Penning, A.T. DinkovaKostova, J.D. Hayes, Mechanisms of activation of the transcription factor Nrf2 by redox stressors, nutrient cues, and energy status and the pathways through which it attenuates degenerative disease, Free Radic. Biol. Med. 88 (2015) $108-146$.

[3] T.W. Kensler, N. Wakabayashi, S. Biswal, Cell survival responses to environmental stresses via the Keap1-Nrf2-ARE pathway, Annu. Rev. Pharmacol. Toxicol. 47 (2007) 89-116.

[4] A. Giudice, C. Arra, M.C. Turco, Review of molecular mechanisms involved in the activation of the Nrf2-ARE signaling pathway by chemopreventive agents, Methods Mol. Biol. 647 (2010) 37-74.

[5] M.C. Jaramillo, D.D. Zhang, The emerging role of the Nrf2-Keap1 signaling pathway in cancer, Genes Dev. 27 (2013) 2179-2191.

[6] A.K. Jaiswal, Nrf2 signaling in coordinated activation of antioxidant gene expression, Free Radic. Biol. Med. 36 (2004) 1199-1207.

[7] H.M. Leinonen, E. Kansanen, P. Polonen, M. Heinaniemi, A.L. Levonen, Role of the Keap1-Nrf2 pathway in cancer, Adv. Cancer Res. 122 (2014) 281-320.

[8] P. Canning, F.J. Sorrell, A.N. Bullock, Structural basis of Keap1 interactions with Nrf2, Free Radic. Biol. Med. 88 (2015) 101-107.
[9] A. Kobayashi, M.I. Kang, H. Okawa, M. Ohtsuji, Y. Zenke, T. Chiba, K. Igarashi, M. Yamamoto, Oxidative stress sensor Keap1 functions as an adaptor for Cul3based E3 ligase to regulate for proteasomal degradation of Nrf2, Mol. Cell. Biol. 24 (2004) 7130-7139.

[10] K. Itoh, N. Wakabayashi, Y. Katoh, T. Ishii, K. Igarashi, J.D. Engel, M. Yamamoto, Keap1 represses nuclear activation of antioxidant responsive elements by Nrf2 through binding to the amino-terminal Neh2 domain, Gene Dev. 13 (1999) $76-86$.

[11] R. Zhao, Y. Hou, Q. Zhang, C.G. Woods, P. Xue, J. Fu, K. Yarborough, D. Guan, M. E. Andersen, J. Pi, Cross-regulations among NRFs and KEAP1 and effects of their silencing on arsenic-induced antioxidant response and cytotoxicity in human keratinocytes, Environ. Health Perspect. 120 (2012) 583-589.

[12] H. Motohashi, M. Yamamoto, Nrf2-Keap1 defines a physiologically important stress response mechanism, Trends Mol. Med. 10 (2004) 549-557.

[13] T.W. Kensler, N. Wakabayash, S. Biswal, Cell survival responses to environmental stresses via the Keap1-Nrf2-ARE pathway, Annu. Rev. Pharmacol. Toxicol. 47 (2007) 89-116.

[14] E.J. Cordova, A. Martinez-Hernandez, L. Uribe-Figueroa, F. Centeno, M. Morales-Marin, H. Koneru, M.A. Coleman, L. Orozco, The NRF2-KEAP1 pathway is an early responsive gene network in arsenic exposed lymphoblastoid cells, Plos One 9 (2014).

[15] P.D. Ray, B.W. Huang, Y. Tsuji, Coordinated regulation of Nrf2 and histone H3 serine 10 phosphorylation in arsenite-activated transcription of the human heme oxygenase-1 gene, Biochim. Et. Biophys. Acta 1849 (2015) 1277-1288.

[16] R. Zhao, B. Yang, L. Wang, P. Xue, B. Deng, G. Zhang, S. Jiang, M. Zhang, M. Liu J. Pi, D. Guan, Curcumin protects human keratinocytes against inorganic arsenite-induced acute cytotoxicity through an NRF2-dependent mechanism, Oxid. Med. Cell. Longev. (2013) 412576.2013.

[17] S. Kurinna, S. Werner, NRF2 and microRNAs: new but awaited relations, Biochem. Soc. Trans. 43 (2015) 595-601.

[18] N.M. Shah, S.A. Rushworth, M.Y. Murray, K.M. Bowles, D.J. MacEwan, Understanding the role of NRF2-regulated miRNAs in human malignancies, Oncotarget 4 (2013) 1130-1142.

[19] R.F. Paulson, L. Shi, D.C. Wu, Stress erythropoiesis: new signals and new stress progenitor cells, Curr. Opin. Hematol. 18 (2011) 139-145.

[20] A. Kim, E. Nemeth, New insights into iron regulation and erythropoiesis, Curr. Opin. Hematol. 22 (2015) 199-205.

[21] A. Kruse, D.E. Uehlinger, F. Gotch, P. Kotanko, N.W. Levin, Red blood cell lifespan, erythropoiesis and hemoglobin control, Contrib. Nephrol. 161 (2008) 247-254.

[22] R.N.V.S. Suragani, R.S. Zachariah, J.G. Velazquez, S.J. Liu, C.W. Sun, T.M. Townes, J.J. Chen, Heme-regulated eIF2 alpha kinase activated Atf4 signaling pathway in oxidative stress and erythropoiesis, Blood 119 (2012) 5276-5284.

[23] H.M. Rogers, X.B. Yu, J. Wen, R. Smith, E. Fibach, C.T. Noguchi, Hypoxia alters progression of the erythroid program, Exp. Hematol. 36 (2008) 17-27.

[24] L.X. Wang, X.Y. Wang, S.P. Zhang, G.B. Qu, S.J. Liu, A protective role of hemeregulated eIF2 alpha kinase in cadmium-induced toxicity in erythroid cells, Food Chem. Toxicol. 62 (2013) 880-891.

[25] X.Y. Wang, L.X. Wang, S.J. Liu, Heme-regulated elF2 alpha kinase plays a crucial role in protecting erythroid cells against Pb-induced hemolytic stress, Chem. Res. Toxicol. 28 (2015) 460-469.

[26] S.A. Peslak, J. Wenger, J.C. Bemis, P.D. Kingsley, J.M. Frame, A.D. Koniski, Y. Chen, J.P. Williams, K.E. McGrath, S.D. Dertinger, J. Palis, Sublethal radiation injury uncovers a functional transition during erythroid maturation, Exp. Hematol. 39 (2011) 434-445.

[27] Y. Kawatani, T. Suzuki, R. Shimizu, V.P. Kelly, M. Yamamoto, Nrf2 and selenoproteins are essential for maintaining oxidative homeostasis in erythrocytes and protecting against hemolytic anemia, Blood 117 (2011) 986-996.

[28] D.T. Rutkowski, R.J. Kaufman, All roads lead to ATF4, Dev. Cell 4 (2003) $442-444$.

[29] M.S. Kilberg, J.X. Shan, N. Su, ATF4-dependent transcription mediates signaling of amino acid limitation, Trends Endocrinol. Metab. 20 (2009) 436-443.

[30] T. Afonyushkin, O.V. Oskolkova, M. Philippova, T.J. Resink, P. Erne, B.R. Binder V.N. Bochkov, Oxidized phospholipids regulate expression of ATF4 and VEGF in endothelial cells via NRF2-dependent mechanism: novel point of convergence between electrophilic and unfolded protein stress pathways, Arter. Thromb. Vasc. 30 (2010) (1007-U275).

[31] M.A. Listowski, E. Heger, D.M. Boguslawska, B. Machnicka, K. Kuliczkowski, J. Leluk, A.F. Sikorski, microRNAs: fine tuning of erythropoiesis, Cell. Mol. Biol. Lett. 18 (2013) 34-46.

[32] C.H. Lawrie, microRNA expression in erythropoiesis and erythroid disorders, Br. J. Haematol. 150 (2010) 144-151.

[33] N. Bianchi, C. Zuccato, A. Finotti, I. Lampronti, M. Borgatti, R. Gambari, Involvement of miRNA in erythroid differentiation, Epigenomics-Uk 4 (2012) 51-65.

[34] Z. Sun, Y. Wang, X. Han, X. Zhao, Y. Peng, Y. Li, M. Peng, J. Song, K. Wu, S. Sun, W. Zhou, B. Qi, C. Zhou, H. Chen, X. An, J. Liu, miR-150 inhibits terminal erythroid proliferation and differentiation, Oncotarget (2015).

[35] D. Yu, C.O. dos Santos, G. Zhao, J. Jiang, J.D. Amigo, E. Khandros, L.C. Dore, Y. Yao, J. D’Souza, Z. Zhang, S. Ghaffari, J. Choi, S. Friend, W. Tong, J.S. Orange, B. H. Paw, M.J. Weiss, miR-451 protects against erythroid oxidant stress by repressing 14-3-3zeta, Genes Dev. 24 (2010) 1620-1633.

[36] E. Penna, F. Orso, D. Taverna, miR-214 as a key hub that controls cancer networks: small player, multiple functions, J. Investig. Dermatol. 135 (2015) 960-969.

[37] T. Sharma, R. Hamilton, C.C. Mandal, miR-214: a potential biomarker and 
therapeutic for different cancers, Future Oncol. 11 (2015) 349-363.

[38] X.G. Wang, B.S. Guo, O. Li, J. Peng, Z.J. Yang, A.Y. Wang, D. Li, Z.B. Hou, K. Lv, G. H. Kan, H.Q. Cao, H. Wu, J.P. Song, X.H. Pan, Q. Sun, S.K. Ling, Y.H. Li, M. Zhu, P. F. Zhang, S.L. Peng, X.Q. Xie, T. Tang, A. Hong, Z.X. Bian, Y.Q. Bai, A.P. Lu, Y.H. Li, F.C. He, G. Zhang, Y.X. Li, miR-214 targets ATF4 to inhibit bone formation, Nat Med. 19 (2013) 93-100.

[39] W.W. Zheng, X.M. Dong, R.H. Yin, F.F. Xu, H.M. Ning, M.J. Zhang, C.W. Xu, Y. Yang, Y.L. Ding, Z.D. Wang, W.B. Zhao, L.J. Tang, H. Chen, X.H. Wang, Y. Q. Zhan, M. Yu, C.H. Ge, C.Y. Li, X.M. Yang, EDAG positively regulates erythroid differentiation and modifies GATA1 acetylation through recruiting p300, Stem Cells 32 (2014) 2278-2289.

[40] Y. Chen, Z. Wang, M. Xu, X. Wang, R. Liu, Q. Liu, Z.H. Zhang, T. Xia, J.C. Zhao, G. B. Jiang, Y. Xu, S.J. Liu, Nanosilver incurs an adaptive shunt of energy metabolism mode to glycolysis in tumor and nontumor cells, Acs Nano 8 (2014) 5813-5825.

41] M. Garcia-Barros, F. Paris, C. Cordon-Cardo, D. Lyden, S. Rafii, A. HaimovitzFriedman, Z. Fuks, R. Kolesnick, Tumor response to radiotherapy regulated by endothelial cell apoptosis, Science 300 (2003) 1155-1159.

[42] C. Hartley, S. Elliott, C.G. Begley, P. McElroy, W. Sutherland, R. Khaja, A. C. Heatherington, T. Graves, H. Schultz, J. Del Castillo, G. Molineux, Kinetics of haematopoietic recovery after dose-intensive chemo/radiotherapy in mice: optimized erythroid support with darbepoetin alpha, Br. J. Haematol. 122 (2003) 623-636.

[43] R.S. Britton, K.L. Leicester, B.R. Bacon, Iron toxicity and chelation therapy, Int. J. Hematol. 76 (2002) 219-228.

[44] W.Y. Lu, M.F. Zhao, S. Rajbhandary, F. Xie, X. Chai, J. Mu, J.X. Meng, Y.J. Liu, Y. Jiang, X.N. Xu, A.M. Meng, Free iron catalyzes oxidative damage to hematopoietic cells/mesenchymal stem cells in vitro and suppresses hematopoiesis in iron overload patients, Eur. J. Haematol. 91 (2013) 249-261.

[45] H.L. Hu, R.A. Gatti, MicroRNAs: new players in the DNA damage response, J. Mol. Cell Biol. 3 (2011) 151-158.

[46] I.A. Babar, F.J. Slack, J.B. Weidhaas, miRNA modulation of the cellular stress response, Future Oncol. 4 (2008) 289-298.

[47] C.E. Cross, M.F. Tolba, C.M. Rondelli, M.X. Xu, S.Z. Abdel-Rahman, Oxidative stress alters miRNA and gene expression profiles in villous first trimester trophoblasts, Biomed. Res. Int. (2015).

[48] N.L. Simone, B.P. Soule, D. Ly, A.D. Saleh, J.E. Savage, W. DeGraff, J. Cook, C. C. Harris, D. Gius, J.B. Mitchell, Ionizing Radiation-Induced Oxidative Stress Alters miRNA Expression, Plos. One 4 (2009).

[49] S. Claro, M.E.M. Oshiro, R.A. Mortara, E.J. Paredes-Gamero, G.J.S. Pereira, S. S. Smaili, A.T. Ferreira, gamma-Rays-generated ROS induce apoptosis via mitochondrial and cell cycle alteration in smooth muscle cells, Int. J. Radiat. Biol. 90 (2014) 914-927.

[50] T.F. Zhuang, H.J. Han, Z.Y. Yang, Iron, oxidative stress and gestational diabetes, Nutrients 6 (2014) 3968-3980.

[51] K. Taoka, K. Kumano, F. Nakamura, M. Hosoi, S. Goyama, Y. Imai, A. Hangaishi, M. Kurokawa, The effect of iron overload and chelation on erythroid differentiation, Int. J. Hematol. 95 (2012) 149-159.

[52] Y. Xu, K.L. Swartz, K.T. Siu, M. Bhattacharyya, A.C. Minella, Fbw7-dependent cyclin E regulation ensures terminal maturation of bone marrow erythroid cells by restraining oxidative metabolism, Oncogene 33 (2014) 3161-3171.

[53] A.K. Jaiswal, Nrf2 signaling in oxidative stress and cell survival, Free Radic. Biol. Med. 45 (2008) (S3-S3).

[54] X.J. Wang, Z. Sun, W. Chen, K.E. Eblin, J.A. Gandolfi, D.D. Zhang, Nrf2 protects human bladder urothelial cells from arsenite and monomethylarsonous acid toxicity, Toxicol. Appl. Pharm. 225 (2007) 206-213.

[55] M. Yu, H. Li, Q. Liu, F. Liu, L. Tang, C. Li, Y. Yuan, Y. Zhan, W. Xu, W. Li, H. Chen, C. Ge, J. Wang, X. Yang, Nuclear factor p65 interacts with Keap1 to repress the Nrf2-ARE pathway, Cell Signal. 23 (2011) 883-892.

[56] H.P. Harding, Y.H. Zhang, H.Q. Zeng, I. Novoa, P.D. Lu, M. Calfon, N. Sadri, C. Yun, B. Popko, R. Paules, D.F. Stojdl, J.C. Bell, T. Hettmann, J.M. Leiden, D. Ron, An integrated stress response regulates amino acid metabolism and resistance to oxidative stress, Mol. Cell 11 (2003) 619-633.

[57] M.X. Bi, C. Naczki, M. Koritzinsky, D. Fels, J. Blais, N.P. Hu, H. Harding, I. Novoa, M. Varia, J. Raleigh, D. Scheuner, R.J. Kaufman, J. Bell, D. Ron, B.G. Wouters, C. Koumenis, ER stress-regulated translation increases tolerance to extreme hypoxia and promotes tumor growth, Embo J. 24 (2005) 3470-3481.

[58] J. Seo, E.S. Fortuno, J.M. Suh, D. Stenesen, W. Tang, E.J. Parks, C.M. Adams, T. Townes, J.M. Graff, Atf4 regulates obesity, glucose homeostasis, and energy expenditure, Diabetes 58 (2009) 2565-2573.

[59] H. Fung, P. Liu, B. Demple, ATF4-dependent oxidative induction of the DNA repair enzyme Ape1 counteracts arsenite cytotoxicity and suppresses arsenite-mediated mutagenesis, Mol. Cell. Biol. 27 (2007) 8834-8847.

[60] A.H. Juan, R.M. Kumar, J.G. Marx, R.A. Young, V. Sartorelli, Mir-214-dependent regulation of the polycomb protein ezh2 in skeletal muscle and embryonic stem cells, Mol. Cell 36 (2009) 61-74.

[61] R. Margueron, D. Reinberg, The polycomb complex PRC2 and its mark in life, Nature 469 (2011) 343-349.

[62] C.J. Chang, M.C. Hung, The role of EZH2 in tumour progression, Br. J. Cancer 106 (2012) 243-247.

[63] D.C.S. Huang, A. Strasser, BH3-only proteins-essential initiators of apoptotic cell death, Cell 103 (2000) 839-842.

[64] M. Ridinger-Saison, E. Evanno, I. Gallais, P. Rimmele, D. Selimoglu-Buet, E. Sapharikas, F. Moreau-Gachelin, C. Guillouf, Epigenetic silencing of Bim transcription by Spi-1/PU.1 promotes apoptosis resistance in leukaemia, Cell Death Differ. 20 (2013) 1268-1278.

[65] K.D. Rasmussen, S. Simmini, C. Abreu-Goodger, N. Bartonicek, M. Di Giacomo, D. Bilbao-Cortes, R. Horos, M. Von Lindern, A.J. Enright, D. O'Carroll, The miR144/451 locus is required for erythroid homeostasis, J. Exp. Med. 207 (2010) 1351-1358.

[66] S. Ghaffari, Oxidative stress in the regulation of normal and neoplastic hematopoiesis, Antioxid. Redox Signal. 10 (2008) 1923-1940.

[67] L. Kautz, E. Nemeth, Molecular liaisons between erythropoiesis and iron metabolism, Blood 124 (2014) 479-482.

[68] Y.Y. Zhu, G.C. Yu, Y. Zhang, Z. Xu, Y.Q. Wang, G.R. Yan, Q.Y. He, A novel andrographolide derivative AL-1 exerts its cytotoxicity on K562 cells through a ROS-dependent mechanism, Proteomics 13 (2013) 169-178.

[69] A. Leecharoenkiat, T. Wannatung, P. Lithanatudom, S. Svasti, S. Fucharoen, D. Chokchaichamnankit, C. Srisomsap, D.R. Smith, Increased oxidative metabolism is associated with erythroid precursor expansion in beta( 0 )-thalassaemia/Hb E disease, Blood Cells Mol. Dis. 47 (2011) 143-157.

[70] L. Lu, A.P. Han, J.J. Chen, Translation initiation control by heme-regulated eukaryotic initiation factor 2alpha kinase in erythroid cells under cytoplasmic stresses, Mol. Cell. Biol. 21 (2001) 7971-7980.

[71] M.R. Campbell, M. Karaca, K.N. Adamski, B.N. Chorley, X. Wang, D.A. Bell, Novel hematopoietic target genes in the NRF2-mediated transcriptional pathway, Oxid. Med. Cell. Longev. (2013) 120305.2013.

[72] C.H. He, P.F. Gong, B. Hu, D. Stewart, M.E. Choi, A.M.K. Choi, J. Alam, Identification of activating transcription factor 4 (ATF4) as an Nrf2-interacting protein - implication for heme oxygenase-1 gene regulation, J. Biol. Chem. 276 (2001) $20858-20865$.

[73] J.A. Simon, C.A. Lange, Roles of the EZH2 histone methyltransferase in cancer epigenetics, Mutat. Res.-Fund. Mol. M 647 (2008) 21-29.

[74] J. Xu, Z. Shao, D. Li, H. Xie, W. Kim, J. Huang, J.E. Taylor, L. Pinello, K. Glass, J. D. Jaffe, G.C. Yuan, S.H. Orkin, Developmental control of polycomb subunit composition by GATA factors mediates a switch to non-canonical functions, Mol. Cell 57 (2015) 304-316.

[75] T. Fujiwara, H. Saitoh, A. Inoue, M. Kobayashi, Y. Okitsu, Y. Katsuoka, N. Fukuhara, Y. Onishi, K. Ishizawa, R. Ichinohasama, H. Harigae, 3-Deazaneplanocin A (DZNep), an inhibitor of S-adenosylmethionine-dependent methyltransferase, promotes erythroid differentiation, J. Biol. Chem. 289 (2014) $8121-8134$

[76] J.M. Adams, S. Cory, The Bcl-2 apoptotic switch in cancer development and therapy, Oncogene 26 (2007) 1324-1337.

[77] D.C. Huang, A. Strasser, BH3-only proteins-essential initiators of apoptotic cell death, Cell 103 (2000) 839-842.

[78] M. Koulnis, E. Porpiglia, P.A. Porpiglia, Y. Liu, K. Hallstrom, D. Hidalgo, M. Socolovsky, Contrasting dynamic responses in vivo of the Bcl-xL and Bim erythropoietic survival pathways, Blood 119 (2012) 1228-1239.

[79] R.M. Abutin, J. Chen, T.K. Lung, J.A. Lloyd, S.T. Sawyer, H. Harada, Erythropoietin-induced phosphorylation/degradation of BIM contributes to survival of erythroid cells, Exp. Hematol. 37 (2009) 151-158. 